\title{
Luces en la noche: sustentabilidad, la ciudad desde un horizonte histórico y la comunicación cinematográfica
}

\section{Lights at night: Sustainability, the city from a historical horizon and cinematographic communication}

Rafael Tonatiuh Ramírez Beltrán*

Universidad Anáhuac México rramirez@anahuac.mx

https://orcid.org/0000-0002-1968-9755

Av. Universidad Anáhuac núm. 46, Col. Lomas Anáhuac,

Huixquilucan, Estado de México, C.P. 052786, México

Editor: Rogelio del Prado Flores

Fecha de recepción: 17 de junio de 2021

https://doi.org/10.36105/stx.2021edesp50aniv.03

Fecha de aceptación: 24 de agosto de 2021

\section{RESUMEN}

El artículo se escribió para conmemorar los 50 años de la Facultad de Comunicación de la Universidad Anáhuac y comienza comparando a ésta con la trayectoria de una ciudad moderna. Se da cuenta de algunos síntomas de la crisis ambiental (cambio climático, crisis sanitaria, deterioro ambiental de los ecosistemas, etc.), para proponer la construcción social colectiva y perenne de la sustentabilidad, revisando dos modelos que han sintetizado sus tendencias teóricas y prácticas. Se documenta las estructuras históricas de las ciudades y se propone seguir haciendo políticas públicas para la sustentabilidad. Finalmente, se recuperan algunos productos comunicativos cinematográficos que problematizan la ciudad y el ambiente. Se llega a conclusiones sobre acciones civiles y gubernamentales a favor de lo sustentable.

Palabras clave: crisis civilizatoria, ciudad y sustentabilidad, evolución de la ciudad, cine ambiental.

\footnotetext{
* Profesor investigador de la Universidad Anáhuac México, adscrito al Centro de Investigación para la Comunicación Aplicada de la Facultad de Comunicación de la Universidad Anáhuac México, Campus Norte, en el que coordina la línea de investigación: Realidad social, comunicación, políticas públicas y tecnología y Vinculación. Pertenece al Sistema Nacional de Investigadores, Nivel I, del Conacyt.
} 


\section{ABSTRACT}

The article was written to commemorate the 50 years of the Faculty of Communication of the Anahuac University and begins by comparing it with the trajectory of a modern city. It realizes some symptoms of the environmental crisis (climate change, health crisis, environmental deterioration of ecosystems, etc.), to propose the collective and perennial social construction of sustainability, reviewing two models that have synthesized their theoretical and practical tendencies. The historical structures of cities are documented, and it is proposed to continue making public policies for sustainability. Finally, some cinematographic communicative products are recovered that problematize the city and the environment. Conclusions are reached on civil and governmental actions in favor of sustainability.

Keywords: civilizing crisis, city and sustainability, evolution of the city environmental, cinema.

\section{INTRODUCCIÓN}

Es un inmenso gusto poder participar con este artículo que se realiza en el marco de las conmemoraciones del medio siglo de la Facultad de Comunicación de la Universidad Anáhuac México.

La historia de la facultad de una institución de educación superior puede compararse a la de la estructura e intensidad evolutiva de una ciudad. Como las urbes, las universidades tienen un momento de surgimiento. El nacimiento de la Licenciatura de Comunicación es el inicio de esta historia en la década de los años setenta del siglo pasado. La licenciatura sigue siendo -en su continua transformación- el núcleo (o el centro) de toda la actividad académica, pero que ahora ya tiene un programa académico hermano que crece todos los años, nos referimos a la Dirección de Entretenimiento.

Como las ciudades, con el crecimiento y desarrollo, la Facultad comenzó una expansión a lo largo y en forma vertical, en los posgrados (especializaciones y maestrías), en los noventa del siglo pasado y se extendió a lo ancho, con un vigoroso programa de extensión de diplomados y cursos. Ya en el siglo XXI, la facultad alcanzó el estatus de metrópoli- facultad, con dos doctorados y un Centro de Investigación para la Comunicación Aplicada.

Hoy, la Facultad es una compleja comunidad perfectamente posicionada e instalada en una sociedad de la información y el conocimiento, que se renueva e innova, año con año, y que tiene clara sus vocaciones como formadora de talento en el campo de la comunicación, la cultura y el entrenamiento, y un compromiso irrenunciable hacia la generación y difusión de nuevo 
conocimiento en el campo de la comunicación. La vida cotidiana de esta Facultad es como las ciudades: imparable, luminosa, compleja y de constante aprendizaje. Se convierte en otra cosa constantemente: a veces es colmena de estudiantes, otra vez es cátedra, estancia, convivencia, taller, investigación, lazo, coloquio, construcción e imperceptible y perenne transformación.

El texto que ahora comparto se instala en la línea en que me desarrollo académicamente y tiene que ver con lo que creemos es el destino inevitable de la humanidad: la sustentabilidad, en el que hay que trabajar si queremos preservar nuestra especie por más largo tiempo entre las que tiene vida en nuestro planeta. Tal vez el espacio de trabajo más complejo para las propuestas y prácticas sustentables sea la ciudad, pero la construcción debe pasar inevitablemente por el repensar los espacios urbanos en forma inevitable, dado que es el sistema artificial construido en el que habita la mayor cantidad de población humana en el mundo.

Hemos sostenido en otros trabajos (Ramírez y Arévalo, 2017) que el crecimiento social y la toma de conciencia a escala planetaria por el concepto sustentabilidad es innegable y su potencial vigoroso en las últimas décadas. Pero también que con frecuencia se ha sesgado, sobreutilizado o tergiversado esta idea, analizando solo una parcialidad de lo que implica el tamaño de la empresa, de lo que significa la transformación social, racional y cultural necesaria para subsistencia humana en los años por venir. La sustentabilidad no es un punto de llegada, sino una edificación humana de otro estilo de civilización hacia el que debemos avanzar, respetando la capacidad de carga de los ecosistemas.

Por lo que esta discusión la vinculamos esta vez a dos temas, articulados entre sí, que consideramos particularmente importantes, a saber: las ciudades en su trayecto histórico y la comunicación de la sustentabilidad en el cine. Lo anterior por dos razones básicas: la tendencia histórica ya irreversible de la ciudad como el lugar en el que vive el mayor número de habitantes de la especie humana en planeta ${ }^{1}$. Y el cine, a pesar de los cambios que ha experimentado en su proyección -de las pantallas en salas cinematográficas a plataformas y dispositivos móviles-sigue teniendo una presencia e influencia importante en las audiencias del mundo que se contabilizan por millones.

Es inevitable referir que la ciudad, las escuelas y el cine a escala planetaria mutaron en 2020/2I, el encierro social durante la pandemia cambió su rostro del bullicio, tráfico y a veces la algarabía al silencio y el vacío en salas, aulas y calles.

En este contexto nos inquieta responder, en este capítulo, algunos cuestionamientos: ¿Son las ciudades espacios habitables en donde es posible la sustentabilidad? ¿El cine está

I En el 2014 la Organización de las Naciones Unidas (ONU) informó que más de la mitad de los 7000 millones de habitantes del mundo vive en áreas urbanas. El organismo señaló que el $54 \%$ de la población mundial vive en ciudades y que para el año 2050 la cifra será de 66\% (ONU, 20I4). Crece población urbana en el mundo (Voz de América, 2019). 
tocando, con pertinencia y suficiencia, temas de sustentabilidad? ¿El cine contemporáneo, como poderosa herramienta de la representación social retrata, propone o visualiza una ciudad sustentable?

\section{DESARROLLO}

\section{Elementos contextuales de la crisis ambiental y la sustentabilidad como cimiento y horizonte}

En los últimos años hemos sido testigos del incremento de los síntomas de una crisis ambiental planetaria innegable, provocada por un estilo de desarrollo absolutamente insustentable. La pérdida de biodiversidad, el deterioro generalizado de los ecosistemas terrestres y marinos, la sobreexplotación de los cuerpos de agua, la pérdida de calidad de aire en a la atmósfera, la erosión de suelos, la pobreza y violencia humana, etc. También la pandemia de la COVID-I9 demostró que la crisis sanitaria es también ambiental y que la especie humana es vulnerable como cualquier otra especie viva.

Otro síntoma de los más evidentes de esta crisis civilizatoria es el relacionado con el cambio climático. El Grupo Intergubernamental de Expertos sobre el Cambio Climático (IPCC, por sus siglas en ingles) en su Informe del 20I8, aseguraba categóricamente:

Durante la última década se ha producido una serie sin precedentes de tormentas, incendios forestales, sequías, blanqueamiento de corales, olas de calor e inundaciones en todo el mundo con solo un grado Celsius de calentamiento global. Pero la situación empeorará con un calentamiento de I,5 grados Celsius. (IPCC, 20I8, p. 4)

Aunque existe evidencia científica de que el cambio climático es el mayor problema que enfrenta la humanidad en la era actual (IPCC, 20I4), hay otros problemas asociados o que se combinan como la pérdida de biodiversidad. En otro informe de las Naciones Unidas (2019) se asegura que:

Los humanos están transformando los paisajes naturales de la Tierra de manera tan dramática que hasta un número tan grande como un millón de especies vegetales y animales están en peligro de extinción, lo que representa una amenaza grave a los ecosistemas (...) existe un declive de la biodiversidad en todo el planeta y a los peligros que crea para la civilización humana. En la mayoría de los principales hábitats terrestres, desde las sabanas de África hasta las selvas de 
Sudamérica, la abundancia promedio de la vida vegetal y animal nativa ha decaído un 20 por ciento o más, principalmente durante el siglo pasado. Con una población mundial de más de siete mil millones de habitantes, las actividades como la agricultura, la tala, la caza ilegal, la pesca y la minería están alterando el mundo natural a un ritmo sin precedentes en la historia de la humanidad. (IPEBS, 2019, p. 2)

Añejos problemas como la perdida de cubierta vegetal, la contaminación atmosférica en las grandes ciudades, la migración, el deterioro en la calidad de vida generalizada, epidemias presentan nuevos rostros agravados por el deterioro generalizado en el mundo.

Es significativo este avance del deterioro ambiental, convertido en una crisis que evidencia una civilización con cimientos endebles, que no parece entender la gravedad del problema.

Desde 1983, en forma más decidida los organismos internacionales (ONU, Unesco, PNUMA, etc.) lideraron la construcción de una alternativa colectiva, que genéricamente se conoció como desarrollo sustentable. La historia se ha contado muchas veces:

El año 1983 de la Comisión de Desarrollo y Medio Ambiente, integrada por un grupo de personalidades del ámbito científico, político y social, representativo de los diversos intereses existentes en la comunidad internacional, El primer concepto que emanó de esa Comisión ya como el Informe Brundtland en 1987, fue asegurar que el desarrollo sea sostenible, es decir, asegurar que satisfaga las necesidades del presente sin comprometer la capacidad de las futuras generaciones para satisfacer las propias (Cortés y Peña, 2015, p. 45).

Ambicioso y vago, simultáneamente, este primer concepto de desarrollo sostenible. Después manipulado de múltiples formas: desde campañas políticas, hasta suavizado en las agencias internacionales o usado como valor agregado de algunas marcas, mercancías y productos.

Se fue imponiendo, desde los noventa del siglo pasado, como un concepto más de base social: la sustentabilidad del desarrollo o simplemente sustentabilidad. Que, en un contexto de crisis ambiental agravada, dejó de ser un concepto aislado en el mundo de la ciencia y las organizaciones políticas, civiles empresariales e internacionales, para alcanzar un estatus pleno en una amplia sociedad civil que sigue constituyendo la base para un debate sobre el futuro de la especie humana en el plano mundial.

Es un concepto, inacabado necesariamente. Algunos autores sostienen que la producción académica que está generando en las ciencias de la sustentabilidad puede ser considerado un boom por su crecimiento y expansión en todo el saber científico. ${ }^{2}$

${ }^{2}$ Víctor Toledo (2015), que fue secretario del Medio Ambiente y Recursos Naturales en México, documentó al respecto: El boom de la sustentabilidad queda certificado por las publicaciones registradas. El volumen de la 
En este crecimiento de las ciencias dedicas a la sustentabilidad, Enrique Leff, a lo largo de su obra ha propuesto la construcción de una epistemología ambiental en la que ha considerado en su constitución conceptos orientadores como la racionalidad, la complejidad ambiental, los saberes, la ética y la pedagogía ambiental, que nutren a la sustentabilidad ambiental. En El manifiesto por la vida, por una ética para la sustentabilidad (2002) se resume de la siguiente forma:

El concepto de sustentabilidad se funda en el reconocimiento de los límites y potenciales de la naturaleza, así como la complejidad ambiental, inspirando una nueva comprensión del mundo para enfrentar los desafíos de la humanidad en el tercer milenio. El concepto de sustentabilidad promueve una nueva alianza naturaleza cultura fundando una nueva economía, reorientando los potenciales de la ciencia y la tecnología, y construyendo una nueva cultura política fundada en una ética de la sustentabilidad -en valores, creencias, sentimientos y saberes- que renuevan los sentidos existenciales, los mundos de vida y las formas de habitar el planeta Tierra. (Leff, 2002, p. 4)

Los límites y potencialidades tienen que ver con la capacidad de carga de los ecosistemas, que es el crecimiento máximo de una especie, población o factor económico que se puede mantener por largo tiempo, considerando los ritmos de recuperación y factores de permanencia de cualquier especie viva. La nueva alianza parte del quebranto en el que está la relación sociedad naturaleza en la crisis antes descrita, utilizando los saberes y tecnologías disponibles para construir una cultura que considere lo ambiental en forma totalmente distinta. El concepto también incluye las inevitables las dimensiones (lo económico, lo social y lo ambiental y sus amplias intersecciones), pero va más allá considerando valores, intangibles, subjetividades y sentidos (Cortés y Peña, 2015, p. 42).

En el medio siglo del ambientalismo también se debatieron dos caminos: el largo o el corto: reforma o revolución. Una transformación radical de toda la racionalidad dominante del estilo de desarrollo de la civilización hegemónica se pensaba que sería la alternativa.

Sin embargo, lo que ha ido pasando es convencimiento por las diferentes impactos y transformaciones de los efectos de la crisis ambiental. El camino largo sucede que se ha acortado. La disponibilidad de una gran cantidad -incluidos los energéticos- de recursos está llegando a su límite a un plazo menor que el que se tenía previsto. En el cielo y en la tierra las señales no son positivas y las respuestas tendrán que precipitarse.

literatura producido en este campo que a pesar de su diversidad ha dado lugar a una práctica científica más unificada, es impresionante: más de 37 ००० autores de 174 países habían producido más de 20 o०o documentos entre I974 y 20 IO (Bettencourt y Kaurc 20II, I954I). De acuerdo con Kajikawa et al. (20I4), cada año aparecen I2 000 artículos dedicados al tema. 


\section{LA SUSTENTABILIDAD, UNA ESPIRAL INTERMINABLE DE POSICIONES Y TEORÍAS: LOS IMPACTOS SOCIOAMBIENTALES}

Por lo anterior reconocemos que la sustentabilidad es necesaria, pero en su implementación vive una dispersión de significaciones. En este apartado damos cuenta de la evolución y dispersión del concepto de sustentabilidad, usando dos cuadros. En el Cuadro I, Lucie Sauvé (1996) realizó el ejercicio de concentrar en cuatro grandes grupos las concepciones que se tienen sustentabilidad. Define a continuación dos elementos clave: características definitorias del modelo de desarrollo sustentable y la concepción asociada del ambiente. Por último, le empata a cada una de estas cuatro concepciones el paradigma educativo que se desprende de esta concepción y práctica.

El Cuadro I es ilustrativo porque articula concepciones generales de desarrollo sustentable con prácticas concretas de educación ambiental. Además, pasa por el amplio abanico ideológico de las propuestas neoliberales y la educación vista como capacitación específica, donde el ambiente es un recurso que hay que utilizar y la lógica es de productividad y eficiencia. La educación es entendida en este extremo solo como capacitación, transferencia de información. En el otro extremo encontramos al desarrollo sustentable entendido como autonomía, con economía colectiva de subsistencia, con una concepción ambiental de territorio y lugar de vida y una posición educativa contextual, significativa y comunitaria, con valores y tecnología que recuperan lo tradicional. Existen blancos y negros en las posiciones sobre la sustentabilidad y sus concepciones asociadas, pero también los grises que se podrían seguir recortando y ampliando.

El Cuadro I nos permite resumir lo que está en juego en las posiciones teóricas de la sustentabilidad. En ellos se confrontan diversos posiciones políticas, sociales y económicas y también distintos proyectos de futuro socioambiental.

Dado el crecimiento sobre la preocupación del ambiente, el Cuadro 2 documenta la proliferación de usos y connotaciones que se han hecho del concepto de sustentabilidad. Se encuentran 22 acepciones o prácticas discursivas o concretas con las que se identifica el término, desde las etimológicas, epistemológicas, hasta la de prácticas como la disminución del impacto o la autonomía, así como las acciones de política pública, o como lo están retomando actores sociales, empresariales y religiosos. Es un concepto vivo y dinámico, pero puede llegar a ser poco preciso de seguir esta tendencia de apertura. Lo importante, para los fines del presente texto, es que muchos de los usos no discriminan el ser empleados en ámbitos urbanos o rurales. 


\section{CUADRO 1. CONCEPCIONES Y CARACTERÍSTICAS DE LA SUSTENTABILIDAD: AMBIENTE Y EDUCACIÓN}

\begin{tabular}{|c|c|}
\hline CONCEPCIONES DE DESARROLLO SUSTENTABLE & PRINCIPALES CARACTERÍSTICAS \\
\hline $\begin{array}{l}\text { Desarrollo continuo debido a la innovación tecnológi- } \\
\text { ca y el libre comercio. CRED 0: crecimiento económico } \\
\text { con base en principios neoliberales que resolverán los } \\
\text { problemas sociales. }\end{array}$ & $\begin{array}{l}\text { Productividad y competitividad dentro de una socie- } \\
\text { dad dominada por el mercado; libre comercio a escala } \\
\text { mundial; innovación científica y tecnológica para el } \\
\text { crecimiento económico, control jurídico. }\end{array}$ \\
\hline $\begin{array}{l}\text { Desarrollo continuo debido a la innovación tecnológi- } \\
\text { ca y el libre comercio. CRED 0: crecimiento económico } \\
\text { con base en principios neoliberales que resolverán los } \\
\text { problemas ambientales sociales. }\end{array}$ & $\begin{array}{l}\text { Mercado libre a escala mundial: innovación científi- } \\
\text { cas y tecnológicas para el crecimiento económico; re- } \\
\text { forma de instituciones políticas, económicas y socia- } \\
\text { les: pactos mundiales o regionales amplios, acuerdos, } \\
\text { legislación, etcétera. }\end{array}$ \\
\hline $\begin{array}{l}\text { Desarrollo alternativo. CREDO: solo un cambio global } \\
\text { completo en los valores sociales y las opciones permi- } \\
\text { tirá el desarrollo de comunidades sustentables. }\end{array}$ & $\begin{array}{l}\text { Desarrollo de una economía bioregional: distinción } \\
\text { entre necesidades reales y deseos, reduciendo la } \\
\text { dependencia, incrementando la autonomía, favore- } \\
\text { ciendo recursos renovables, estimulando procesos } \\
\text { democráticos, participación y solidaridad, etcétera. }\end{array}$ \\
\hline $\begin{array}{l}\text { Desarrollo autónomo (indígena). CREDO: el desarro- } \\
\text { llo es valorado y está arraigado en la identidad cultu- } \\
\text { ral y sí preserva su integridad territorial. }\end{array}$ & $\begin{array}{l}\text { Economía colectiva de subsistencia con base en la so- } \\
\text { lidaridad, asociada con el territorio propio y extraída } \\
\text { de una cosmogonía distintiva. }\end{array}$ \\
\hline CONCEPCIÓN DELAMBIENTE ASOCIADA & PARADIGMA EDUCATIVO ASOCIADO \\
\hline $\begin{array}{l}\text { El ambiente entendido como recurso a ser desarro- } \\
\text { llado y manejado; uso racional de los recursos para } \\
\text { una ganancia sustentable y una calidad de vida sus- } \\
\text { tentable. }\end{array}$ & $\begin{array}{l}\text { Paradigma racional: la educación entendida como } \\
\text { capacitación, como un proceso de transferencia de } \\
\text { información (principalmente de naturaleza científica, } \\
\text { tecnológica y jurídica). }\end{array}$ \\
\hline $\begin{array}{l}\text { El ambiente biosférico completo, entendido como un } \\
\text { reservorio de recursos a ser manejados globalmente } \\
\text { por organizaciones cúpula. }\end{array}$ & $\begin{array}{l}\text { Paradigma racional: aproximación parecida a la } \\
\text { concepción previa de desarrollo sustentable, pero } \\
\text { aceptando algunas críticas a las fallas del sistema } \\
\text { neoliberal. }\end{array}$ \\
\hline El ambiente entendido como un proyecto comunitario. & $\begin{array}{l}\text { Paradigma inventivo: proceso de investigación crítica } \\
\text { conducido por la comunidad hacia la transformación } \\
\text { de las realidades sociales. }\end{array}$ \\
\hline $\begin{array}{l}\text { Ambiente entendido como territorio (lugar para vivir) } \\
\text { y como un proyecto cultural comunitario. }\end{array}$ & $\begin{array}{l}\text { Paradigma inventivo: construcción de significantes } \\
\text { contextuales y conocimiento útil, tomando en cuenta } \\
\text { los valores y la tecnología tradicionales. }\end{array}$ \\
\hline
\end{tabular}

FUENTE: SAUVÉ, 2006, P. 28. 


\section{CUADRO 2. DISPERSIÓN DEL CONCEPTO DE SUSTENTABILIDAD}

\begin{tabular}{|c|c|}
\hline 1 & Sustentabilidad, etimológicamente: dominar por debajo. \\
\hline 2 & Desde la base del pensamiento: epistemología de un pensamiento alternativo. \\
\hline 3 & $\begin{array}{l}\text { Desde su propia evolución conceptual: } 45 \text { años de síntesis de elaboración global de los organismos } \\
\text { mundiales, ante la alerta de la crisis. }\end{array}$ \\
\hline 4 & $\begin{array}{l}\text { Desde la economía: cómo sostener en el tiempo, no permitir la caída de un indicador como, por } \\
\text { ejemplo, la rentabilidad. }\end{array}$ \\
\hline 5 & $\begin{array}{l}\text { Desde el mercado: posicionamiento de un producto o servicio, políticamente correcto, hacia la } \\
\text { naturaleza o de branding sustentable. }\end{array}$ \\
\hline 6 & $\begin{array}{l}\text { Como regreso: el retorno a prácticas y concepciones de un mundo ideal olvidado o tradicional } 0 \\
\text { premoderno. }\end{array}$ \\
\hline 7 & $\begin{array}{l}\text { Menor impacto: daño menor al entorno, va de lo limpio a lo eficiente. De elementos como la paz al } \\
\text { ahorro por uso de eco-técnicas. }\end{array}$ \\
\hline 8 & $\begin{array}{l}\text { Autonomía: Io sustentable es la práctica comunitaria de la autonomía con cierto grado de } \\
\text { organización propia y de libertad. }\end{array}$ \\
\hline 9 & Nueva red: de intercambio de saberes, cadenas productivas, comercio justo, etc. \\
\hline 10 & Asociado a la vida: analogías de lo sustentable como equilibrio natural de vida. \\
\hline 11 & Valor social: como diferenciador social; vivienda sustentable. También valor superior. \\
\hline 12 & Otra R: de reusar, reducir, reciclar, ampliado a: resistir, respetar, reparar, rechazar, reclama, religarse. \\
\hline 13 & Forma coercitiva de futuro: restringir, limitar, prohibir, castigar, penalizar como formas de políticas. \\
\hline 14 & Optimización, solución e innovación: creación y producción limpia. \\
\hline 15 & Como alternativa y resistencia: resiliente, frugalidad y antifragilidad. \\
\hline 16 & Vida saludable: vivir en el campo, comer vegetales, hacer meditación, tener vida sana. \\
\hline 17 & $\begin{array}{l}\text { Políticas públicas: áreas naturales, vida silvestre, ecoturismo, impacto ambiental, ordenamiento } \\
\text { territorial. }\end{array}$ \\
\hline 18 & Fe y esperanza en la humanidad: Laudato sí, expresiones espirituales. \\
\hline 19 & Ciudadanía sustentable: diferentes formas de habitar el planeta. \\
\hline 20 & Planeación pública: instituciones, tareas y presupuestos en los tres órdenes de gobierno. \\
\hline 21 & $\begin{array}{l}\text { Como responsabilidad: en las empresas de todos los tamaños (de transnacionales a minipymes) } \\
\text { están incluyendo acciones a favor del ambiente. }\end{array}$ \\
\hline 22 & $\begin{array}{l}\text { Como proyecto comunitario: tejidos horizontales, en su forma extrema anticapitalista. Comunidad } \\
\text { ligada a la naturaleza. }\end{array}$ \\
\hline
\end{tabular}

FUENTE: RAMÍREZ Y ARÉVALO, 2017, P. 26-29. 
HISTORIA DE LA CIUDAD Y SU MODERNA

INSUSTENTABILIDAD: UN ESBOZO

No siempre el ser humano vivió en ciudades. En su largo trayecto evolutivo fue un huésped más de la naturaleza, como los millones de especies vivas, con las que le ha tocado coexistir. Su característica gregaria, por sus limitaciones físicas, hizo del grupo una compañía necesaria, natural y de sobrevivencia. Vivió en clanes unidos por lazos de parentesco y en tribus en las que lo fueron ligando lenguas y costumbres. Pero todavía no tenía un lugar fijo para vivir. Era nómada. Cambiaba constantemente de campamentos. Caminaba. Migraba. Cazaba, comía y huía.

La naturaleza se lo imponía con los cambios de clima, con los limitados recursos, las amenazas de otras especies, las agrestes montañas y cordilleras, con necesidades de pesca $\mathrm{y}$ agua.

Pasaron miles de años antes de que sucediera lo que Gordon Childe llama las tres revoluciones del neolítico (aproximadamente entre 8 ooo y 4 ooo antes de la era cristiana): la primera revolución que transformó a la especie humana fue la de la agricultura; en la que el ser humano comienza a tener control de su propio abastecimiento por medio de la domesticación de animales y plantas. Seleccionar, sembrar, cultivar plantas y hierbas, y domesticar animales fueron acciones que lo fijaron a un territorio. Este paso gigantesco fue la base de la segunda revolución: la del excedente productivo y la construcción y organización de centros urbanos, en los que se generaron intercambios comerciales, formación de clases que dominaban y eran protegidas por funcionarios y guerreros y el desarrollo de muchos saberes prácticos y técnicos que originaron la tercera revolución: la del conocimiento (Gordon Childe, I975, p. 155).

Desde la ciudad Sumeria que fue ciudad -estado, amurallada y cerrado sobre sí misma-, pasando por las ciudades griegas y romanas marcadas por la racionalidad, funcionalidad, armonía y orden, a las de la edad media, el renacimiento y las barrocas, las funciones de la ciudad eran de gobierno, dominio, religiosas y comerciales, cada una determinada por su circunstancia histórica. Sin embargo, el inicio del crecimiento de las ciudades comienza cuando son atractivas para la población.

Desde los tiempos de la ciudad medieval y la renacentista, ha resultado atractiva la residencia en las ciudades, asumidas como centros de interacción de grupos sociales, donde se comercializaban mercancías y tenían lugar actividades económicas, políticas, religiosas, sociales y culturales. Sin embargo, esta preferencia provocó el incremento de la demanda de espacios y recursos naturales, incluyendo el suelo, que en muchas ocasiones pasó a ser un elemento secundario ante el diseño y expansión de la urbe (Soto-Cortés, 2015, p. I3I). 
La ciudad industrial tiene -como se sabe- menos de doscientos años. Es una reconfiguración de las ciudades ya establecidas, por una inmensa transformación productiva. Surge en un modelo que demanda fuerza de trabajo que transforme materia prima en mercancías. Necesita población libre que se pueda desplazar del campo a la ciudad, en forma permanente a las zonas de producción.

La ciudad comienza a jugar un papel fundamental del modelo capitalista, que pretende dominarlo todo: naturaleza, poblaciones, mercados, hábitos, tiempos-movimientos, disciplinas, modelos, costumbres, valores, formas de pensamientos, principios científicos y tecnológicos, formas de transporte y áreas habitacionales. La ciudad, en este imaginario, se vuelve lugar que te salva, gracias a este modelo gratificante, de los peligros y amenazas de tradiciones, la pobreza y la naturaleza.

La ciudad contemporánea es el sitio, el espacio físico que devendrá en espacio construido artificial, en el que la modernidad soñará el bienestar y el fin de los malos tiempos. El progreso llegó y edificó una forma diferente de casa en un nuevo hábitat que transformaba los recursos naturales.

En efecto, nunca en la historia de la humanidad, como en esta época, el ser humano logró mejorar sus niveles de salud (baste ver la expectativa de vida al nacer), de educación, de reproducción de la especie como en los primeros I5O años después de la revolución industrial. Nunca tampoco logró la producción de bienes materiales como en esta época. La ciudad ha sido el pilar básico de este incremento en las condiciones de bienestar y en el incremento de los productos internos brutos, en términos económicos de los países.

Lo que pasó en las ciudades en estos tiempos modernos y posmodernos es del tamaño de las trasformaciones antes citadas del neolítico. Sin embargo, y a diferencia de esas revoluciones sociales, el impacto ambiental de esta era es irreductible, innegable y devastador.

Las ciudades evidencian la crisis ambiental, síntomas de deterioro, polución o contaminación en la atmósfera, el suelo y el agua; problemas en la movilidad y el transporte; residuos domésticos e industriales; contaminación, sonora, lumínica y visual; concentración de la población; disminución de áreas verdes, etcétera.

Las ciudades tienen grandes necesidades de abastecimiento de agua, energía, alimentos, transportación, bienes, servicios, material de construcción, almacenamiento de innumerables productos, etc. Y también manejo de residuos sólidos y de agua que ha sido utilizada en forma industrial y doméstica. Las ciudades demandan seguridad, orden, aire limpio, áreas vedes, zonas educativas, clínicas y hospitales próximos. Las ciudades están reglamentadas por instrumentos de política ambiental, como el riesgo, los ordenamientos territoriales y los manifiestos de impacto ambiental, que difícilmente se cumplen. Necesidades diarias de 
millones de habitantes que desafían el frágil equilibrio social, de consumo y ambiental de las urbes del siglo XXI.

\section{CIUDAD Y SUSTENTABILIDAD: LA BÚSQUEDA}

\section{ACTUAL DE ARMONÍA DE UNA DIFÍCIL RELACIÓN}

Por lo anterior, la relación ciudad-sustentabilidad es endeble, un equilibrio en riesgo de perderse permanentemente. La sustentabilidad antes definida -considerando su dispersiónno es fácil en un equilibrio urbano, siempre a punto de perderse. La ciudad se piensa hoy en la solución de sus problemas; la sustentabilidad, apuesta al mañana. En lógicas inestables no siempre el hoy y el mañana son consecuencia.

Los habitantes de las megalópolis saben que, de la vulnerabilidad cotidiana, en tiempos de cambio climático, los factores de riesgo suelen acumularse, por lo que una lluvia pertinaz durante días puede causar inundaciones; un acto político, criminal o terrorista afecta la dinámica social; la sequía o el estiaje prolongado, puede convertirse en incendios que afectan la calidad del aire; una huelga de un servicio afecta la dinámica urbana; las zonas de pobreza se vuelven con frecuencia estigmatizadas de violentas y peligrosas y se recomienda evitarlas; o en zonas sísmicas o riesgo natural, ante un evento, se puede paralizar la sociedad por semana.

Para reducir el riesgo y lograr una mejor adaptación el Centro de investigación Mario Molina propone caminar hacia ciudades sustentables que tendría que promover esquemas de desarrollo urbano de baja intensidad de carbono con un uso racional de los recursos naturales, particularmente el agua y la energía (Molina, s.f.).

Por lo que la sustentabilidad debe convertirse en un transversal inevitable de las políticas públicas urbanas que haga planeaciones con temporalidades del presente, mediano y largo plazo. En la detección y resolución de problemas ambientales se tendrá que tener una visión de complejidad, trabajo interdisciplinario e integral. La calidad de vida debe ser un bien por todos valorado y ponderar factores como la salud y el mejor aprovechamiento del tiempo de empleo, transportación y ocio, así como una alimentación favorable.

En la última década en las grandes ciudades latinoamericanas (Bogotá, Buenos Aires, Ciudad de México, Montevideo, San José, Santiago, etc.), con sus múltiples contradicciones y limitaciones han comenzado un lento trayecto de acciones ciudadanas: como el regreso al uso de la bicicleta, la generación de huertos urbanos con fines alimenticios, la recuperación y ciudadanizar áreas verdes, deportivas y culturales y el aprovechamiento de los móviles para reinventar las ciudades inteligentes con tecnologías para la sustentabilidad. 
La cultura, la educación y la comunicación ambiental comienzan a cosechar incipientes frutos, en prácticas específicas, pero el camino tendrá que seguir.

\section{COMUNICACIÓN, SUSTENTABILIDAD Y CINE CONTEMPORÁNEO}

En la actualidad, la comunicación permanece asociada a términos como: conexión, diálogo, manifestación, manipulación, mediación cultural o interacción simbólica. La comunicación es el centro neurálgico de los objetivos del desarrollo sustentabley de iniciativas como el buen vivir.

De la investigación en comunicación nos hemos dedicado a algo específico, que se relaciona con lo anterior en forma orgánica: la educación y comunicación ambiental en el cine. Hemos construido seis categorías que nos han orientado en el análisis cinematográfico: estudiamos las películas dedicadas a: la naturaleza, la relación hombre -naturaleza, la relación sociedad-naturaleza, la crisis ambiental, el futro de un mundo apocalíptico y el cine sustentable (Ramírez y Meixueiro, 2017).

Para los fines de este trabajo nos detendremos en el cine que nombramos como de comunicación sustentable, este es: películas que proponen alternativas sociales, culturales, ecológicas y tecnológicas a la crisis ambiental o a la civilizatoria. Señalan visiones, acciones y prácticas distintas. Tienden a recuperar el saber tradicional y las posibilidades alternativas ante la problemática ambiental actual. Un elemento central a seguir es la integración de la ecología, la economía y la sociedad, en términos complejos. Los indicadores a seguir son: los saberes alternos y/o tradicionales de los personajes en relación con la naturaleza; la existencia a los recursos naturales y su aprovechamiento limitado; la crítica que se hace a los modelos insustentables; las estrategias específicas que se promueven; las condiciones de mejora de la visión del futuro (Ramírez y Meixueiro, 2015).

Ejemplos de estas películas son: Los sueños (1990), el cortometraje Binta y la gran idea contenido en la cinta En el mundo a cada rato (2004), Alamar (2009), Wall-e (2008), La abuela grillo (2009), Home (2009), Avatar (2009), La toma (2004), La bistoria de las cosas (2007), México Pelágico (2014).

El cine mundial ha recuperado la sustentabilidad por lo podemos ver ejemplos recientes como: La sal de la Tierra (Alemania-Brasil, 20I4), El capitán Fantástico (EUA, 20I6), El Olivo (España, 2016), Ardor (Argentina, 20I4), Artico (Islandia, 20I8), Mañana (Francia, 2015), en las que encontramos elementos como: la recuperación de la tradición de los ancestros, la lucha por territorialidad, la sustentabilidad como alternativa existencial y también como resistencia y lucha: sociales e individuales y que el futuro sigue siendo una posibilidad sobre todo en ámbitos no urbanos y modernos. 


\section{CONCLUSIONES}

La civilización, con sus promesas de desarrollo, ilimitado, crecimiento y progreso, atraviesa por una crisis grave cada vez más evidente. El límite fue la naturaleza, de la que partió todo. El desarrollo de la humanidad como especie, por y para su propia sobrevivencia tiene que atarse al concepto fuerza de la sustentabilidad. Pero estamos en la meta de salida de un trayecto que se antoja largo y sinuoso. Sobre todo, porque lo sustentable se ha bifurcado como idea, esta ramificación ha permitido pensar pluralmente el presente y el futuro, pero es indispensable consensuar formas correctas.

De igual forma, si se piensa en políticas públicas urbanas desde la creación y diseño hasta la evaluación continua. En el trabajo nos detuvimos a analizar a la ciudad, como el destino en el que ya habitan la mayoría de las personas del mundo, en una tendencia que al parecer no tiene retorno. Sin embargo, las ciudades están lejos de ser espacios sustentables, vivibles y seguros.

No obstante, observamos que comienza a ver luces de sustentabilidad que iluminan con ciudadanías ambientales el paisaje de hierro y concreto. Algunas políticas públicas sobre la movilidad, el uso del agua, el uso de espacios públicos y parques y jardines y el manejo de los residuos sólidos y muchas acciones desde la sociedad civil son luces de sustentabilidad en la mar de la noche de la policrisis.

En el cine referido a la sustentabilidad está produciéndose y creciendo en todo el mundo. Lamentablemente vemos muy poco de estas nuevas ciudadanías ambientales en los mensajes cinematográficos. La ciudad, tal y como la conocemos, no parece ser una opción a la sustentabilidad en la comunicación cinematográfica.

Notamos pues una contradicción: la ciudad es la receptora y hogar de los habitantes del mundo, pero la máquina de los sueños que es el cine (y otros medios de comunicación, incluidas las redes sociodigitales) no la considera viable para proporcionar a sus habitantes la calidad de vida que necesitan.

En el futuro cercano veremos más cine sustentable y tal vez recupere lo que ya pasa con muchos de sus políticas, prácticas e intentos de construir una ciudad alternativa y sustentable.

\section{REFERENCIAS}

Centro Mario Molina. (2019). Ciudades sustentables. http://centromariomolina.org/ciudades-sustentables/

Cortés, G., y Peña, J. (2015). De la sostenibilidad a la sustentabilidad. Modelo de desarrollo sustentable para su implementación en políticas y proyectos. Revista Escuela de Administración de Negocios, 78 , 40-54. 
Gordon-Childe, V. (1975). El origen de la civilización. Fondo de Cultura Económica.

Panel Intergubernamental de Expertos sobre el Cambio Climático. (20I4). Quinto Informe de evaluación sobre impacto adaptación y mitigación de Cambio Climático. https://www.ipcc.ch/sri5/

Panel Intergubernamental de Expertos sobre el Cambio Climático. (20I4). Informe de síntesis. https://www.ipcc.ch/site/assets/uploads/2018/o2/SYR_AR5_FINAL_full_es.pdf

Panel Intergubernamental de Expertos sobre el Cambio Climático. (2018). Special report Global Warming of I. $5^{\circ} \mathrm{C}$. https://www.ipcc.ch/site/assets/uploads/sites/2/20I9/og/IPCC-SpecialReport-I.5-SPM_es.pdf

Leff, E, et. al. (2002). Manifiesto por la vida. Por una Ética para la Sustentabilidad. Ambiente \& Sociedad, (I0), 2-I4.

Organización de las Naciones Unidas. (20I4). Crece población urbana en el mundo. https://www. voanoticias.com/a/reporte-mega-ciudades-problemas/1955186.html

Ramírez, R., y Meixueiro, A. (20I5). Cine y Educación Ambiental. Universidad de Guadalajara.

Ramírez, R., y Arévalo, R. (2017). Comunicación Sustentable y Responsabilidad Social Empresarial. Tirant lo Blanch, pp. 5-45.

Ramírez, R., y Meixueiro A. (2017). La representación de la ciencia en el cine y dos propuestas educativas. En Reyes, J. Castro, E. y Noguera P. La vida como centro arte y educación ambiental (pp. II7-I44). Universidad de Guadalajara; Universidad Nacional de Colombia.

Sauvé, L. (1996). Environmental education and sustainable development: A further appraisal. Canadian Fournal of Environmental Education, I, 7-34.

Sauvé, L. (2006). Educar para el debate. Revista Trayectorias de Ciencias Sociales, 20 y 21, 74-87.

Science y Policy for People and Nature. (20I9). Media Release: Nature's Dangerous Decline 'Unprecedented'; Species Extinction Rates 'Accelerating'. https://www.ipbes.net/news/Media-Release- Global-Assessment

Soto-Cortés, J. (2015). El crecimiento urbano de las ciudades: enfoques desarrollista, autoritario, neoliberal y sustentable. Revista Paradigma económico, 7(I), I27-I49.

Toledo, V.(2015). ¿De qué hablamos cuando hablamos de sustentabilidad? Una propuesta ecológico política. Revista Interdisciplina, 3(7), 35-55.

Voz de América. (20I9). Reporte megaciudades. https://www.voanoticias.com/a/reporte-mega-ciudades-problemas/1955 186. html

Esta obra está bajo Licencia Creative Commons Atribución-NoComercial-SinDerivadas 4.o Internacional. 